\title{
Utilization of Agro-based Adsorbents in Binary Wastewater Treatment
}

\author{
Abhiram Siva Prasad Pamula1, Yung-Tse Hung1,*, Howard H. Paul ${ }^{2}$ \\ ${ }^{1}$ Department of Civil and Environmental Engineering, Cleveland State University, Cleveland, Ohio 44115, USA. \\ ${ }^{2}$ Department of Information Systems, Cleveland State University, Cleveland, Ohio 44115, USA.
}

\section{ART I C LEDETAILS}

\section{Article history:}

Received 14 May 2021

Accepted 27 May 2021

Available online 24 June 2021

\section{Keywords:}

Low-Cost Adsorbents

Crystal Violet

Coffee Wastewater

\begin{abstract}
A B S T R A C T
The application of agro-based adsorbents is growing in the tertiary stage of the wastewater treatment process during the presence of hazardous pollutants. Dye and coffee industries are among the major wastewater pollutant sources negatively affect aquatic ecosystems and human health. The current study attempts to treat a binary mixture of crystal violet (CV) and coffee wastewater using agro-based adsorbents such as peanut hull and onion peel. The performance and efficacy of low-cost adsorbents were evaluated using parameters, including transmittance and non-purgeable organic carbon (NPOC). Batch adsorption studies were conducted to optimize both the adsorbent size and dosage that affect the treatment process. The experimental data obtained from the experiment were analyzed to understand whether Langmuir or Freundlich best fits the treatment process's experimental data. It was observed that Langmuir isotherm seems to fit experimental data using peanut hull and Freundlich isotherm using onion peel. The kinetics of the adsorption process appears to follow the pseudo-first-order kinetic model. The regression coefficient value of onion peel was 0.91 , and uptake was $58.14 \mathrm{mg} / \mathrm{g}$. Similarly, using the peanut hull, the regression coefficient was 0.99 , and uptake was $57.47 \mathrm{mg} / \mathrm{g}$. It seems that peanut hull appears to perform better as a low-cost adsorbent compared to onion peel. The adsorption capacity increased with the increasing dosage of low-cost adsorbent (peanut hull) until the adsorbent size of 0.6-0.425 $\mathrm{mm}$ and steadily decreased after that.
\end{abstract}

\section{Introduction}

Water is an essential natural resource for all biological species and a fundamental requirement for the wellbeing of humans. Pollutants, including dyes, heavy metals, pesticides, and fertilizers, are being released into surface water by land management practices, including agriculture and industries. Industries that produce dyes are a serious problem if they release the untreated wastewater into surface water bodies. Wastewater treatment has been a challenge to environmental engineers due to these synthetic pollutants' complexity. Synthetically produced dyes stay longer in the environment and are usually resistant to conventional biological wastewater treatment methods [1]. Based on several industries' high usage volume, dyes pollute surface water due to their high biological oxygen demand (BOD) and chemical oxygen demand (COD). This impact on water quality can impact all the living organisms, from microbes to humans. Dyes can cause skin-related problems upon physical contact and respiratory illnesses when inhaled [2].

Most importantly, they diminish surface water quality and negatively affect aquatic plants' photosynthesis by reflecting or absorbing most of the sunlight leading to depletion of dissolved oxygen (DO) [3]. This depletion of DO and surge in BOD can cause ecological dead zones in surface water if dye wastewater is untreated. Therefore, dye removal from water is challenging for environmentalists and important to maintain surface water quality. CV dye is used in textile operations and as a biological stain in human and veterinary medicine. Being a stable compound, it has several uses as an antimicrobial agent in animal feeding operations such as poultry. Synthetic manufacturing and stability to biological degradation make CV a potential carcinogen. Previously researchers have found CV frequently causing tumor growth in some aquatic species [4]. An increase in production and usage of this dye can lead to pollution of surface water streams from textile industries, medical facilities, and animal feeding operations.

Conventional technologies involved in dye wastewater treatment include biological wastewater treatment, adsorption, coagulation, membrane filtration, photocatalytic degradation, electrochemical oxidation, and ozonation [5-7]. Based on previous literature, adsorption was the most efficient treatment process to treat hazardous waste in wastewater. Hence to remove $\mathrm{CV}$ in wastewater, adsorption is the treatment process assessed in the current study. But adsorption using activated carbon requires capital and a lot of investment in procuring the material. Also, the spent carbon recycling has its limits on efficiency in the large-scale application of dye wastewater treatment process. Hence there is a need for low-cost adsorbents in preliminary treatment to remove dyes from the aqueous environment. Materials from agricultural waste, which are efficient sorbents, can be used as a potential replacement to the activated carbon $[8,9]$. These materials include peanut hull, onion peel, which can be available in large quantities and inexpensive. Previous literature indicates that binary wastewater consists of both CV and coffee waste was never treated using peanut hull and onion peel. In this research study, low-cost adsorbents, including peanut hull and onion peel, were used to remove combined $\mathrm{CV}$ dye and coffee waste from binary wastewater.<smiles>CN(C)c1ccc(C(=C2C=CC(=[N+](C)C)C=C2)c2ccc(N(C)C)cc2)cc1</smiles>

Fig. 1 Molecular structure of crystal violet (CV) dye 


\subsection{Crystal Violet (CV)}

CV is a tri-phenyl methane dye that has multiple uses in the textile industry and medical applications. The complex structure of the synthetic $\mathrm{CV}$ is as illustrated in Fig. 1. It is predominantly known to be carcinogenic and mutagenic [10]. This dye can be absorbed by the human skin and result in acute toxicity. Long-term exposure to this dye can cause problems, including respiratory illnesses, renal failure, and blindness. The treatment of $\mathrm{CV}$ is a challenge because it is electron-deficient, making it non-biodegradable in both soil and the aquatic environment. Based on all the conventional treatment methods available for hazardous waste treatment, adsorption seems to be an efficient and economical treatment option using low-cost agro-based adsorbents.

\subsection{Coffee Wastewater}

The coffee industry uses a large amount of water for processing in the production process. Based on previous literature, it was estimated that 40 to $45 \mathrm{~L}$ of water is required per $\mathrm{kg}$ of coffee processing [11]. Wastewater produced from the coffee processing industry majorly contains long carbon chains due to caffeine, fat, and other peptic substances. Degrading these long carbon chains takes a long time and using conventional wastewater treatment methods to treat coffee wastewater is difficult. Potential problems caused by the coffee wastewater are depletion of DO due to high BOD and COD [12]. Adsorption based technology using lowcost adsorbents is a potential treatment process that was studied in the current investigation.

\subsection{Onion Peel}

Agro-based adsorbents, including rice husk, peanut hull, onion peel, orange peel, tannin, moringa has a wide range of applications in the treatment and removal of pollutants from the air, water, and wastewater $[13,14]$. Many researchers have recently used onion peel to remove petroleum hydrocarbons from wastewater $[15,16]$. Apart from petroleum hydrocarbons, onion peel was tested as an adsorbent to remove methylene blue (MB) from synthetic wastewater $[17,18]$. Therefore, onion peel was selected and assessed to remove binary CV and coffee from wastewater.

\subsection{Peanut Hull}

The peanut hull has been used as an adsorbent to remove cationic, anionic, and reactive dyes in its raw form [19]. Removing methylene blue (MB) using peanut hull has been extensively studied previously [20, 21]. Since peanut hull is a good bio-sorbent in removing different dyes from wastewater, its efficiency in removing nitrophenyl dye was tested.

\subsection{Activated Carbon}

Activated carbon is one of the most stable, less reactive, and effective adsorbents to remove organic, reactive, and hazardous wastewater pollutants. Although powdered activated carbon can be produced from agricultural wastes, it is not economically feasible, and the cost involved in the reactivation process might be a long-term liability [22].

\section{Experimental Methods}

\subsection{Adsorbate}

$\mathrm{CV}$ certified used in this study was bought from Sigma-Aldrich company and used mixing with processed coffee wastewater. A stock solution of both CV and coffee wastewater with $1000 \mathrm{mg} / \mathrm{L}$ concentration was prepared in the laboratory and diluted when preparing batch samples for the adsorption.

\subsection{Adsorbents}

The adsorbents used in the treatment process include peanut hull and onion peel. Both the peels obtained were washed thoroughly with tap water and air-dried for $48 \mathrm{hrs}$. The dried adsorbents were ground to a fine powder and sieved using 3.327-2.38, 2.38-2.362, 2.362-0.6, 0.6-0.425, $<0.425 \mathrm{~mm}$ sieves. Peanut hull used in the treatment process was used with different sizes at a constant dosage of $1 \mathrm{~g}$. Onion peel used was sieved with a sieve size of $<0.425 \mathrm{~mm}$, and dosages were varied from 0.4 to $2 \mathrm{~g}$.

\subsection{Reference Adsorbent}

Powdered activated carbon (PAC) grade HDC was used as a reference adsorbent in the adsorption process. The manufacturer of the PAC is DARCO. In the current comparative study of transmittance, PAC was used to understand how peanut hull and onion peel works with the pretreatment of combined CV and coffee wastewater.

https://doi.org/10.30799/jespr.213.21070103

\subsection{Categorization of Dye Wastewater Concentration}

Dye wastewater samples of $\mathrm{CV}$ prepared in the laboratory were categorized into three types, such as low, medium, and high concentration. The categorization of concentration in $\mathrm{CV}$ wastewater is tabulated as shown in Table 1.

Table 1 Crystal violet dye and coffee wastewater categorization based on the concentration

\begin{tabular}{ll}
\hline Category & Concentration $(\mathrm{mg} / \mathrm{L})$ \\
\hline Low concentration & 5 \\
Medium concentration & 10 \\
High concentration & 20 \\
\hline
\end{tabular}

\subsection{Batch Experiments}

Adsorption was carried out in centrifuge bottles with a $50 \mathrm{~mL}$ sample with concentrations of 5, 10, and $20 \mathrm{mg} / \mathrm{L}$ of CV. From the coffee stock solution of $1000 \mathrm{mg} / \mathrm{L}$, samples were diluted to $100,150,200,250$, and $300 \mathrm{mg} / \mathrm{L}$. The diluted coffee samples were then mixed with dye wastewater to prepare a binary wastewater solution. Adsorbents including PAC, peanut hull, and onion peel were added and mixed vigorously on a shaker at $100 \mathrm{rpm}$ (fast shake) for 1 minute and $30 \mathrm{rpm}$ (slow shake) for 30 minutes. All the solutions were mixed and adsorbed at a room temperature of $25 \pm 2{ }^{\circ} \mathrm{C}$. After adsorption, the batch samples were filtered using microfiltration, as shown in Fig. 2. The percentage uptake of combined dye and coffee wastewater is as follows:

$$
\text { Percentage Uptake }(\%)=\frac{C_{o}-C_{t}}{C_{o}} * 100
$$

where $C_{0}$ is the initial concentration and $C_{t}$ is the equilibrium concentration at a time $t$.

The binary wastewater concentrations were analyzed using the Carolina UV-VIS spectrophotometer. All the measurements were taken at the wavelength corresponding to the maximum absorption of $\mathrm{CV}$, i.e., $\lambda_{\max }$ $=590 \mathrm{~nm}$.

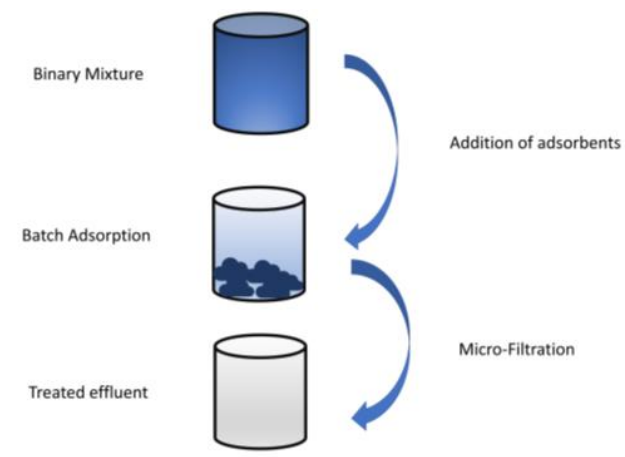

Fig. 2 Process flow diagram of adsorption and microfiltration processes

\section{Results and Discussion}

Factors including adsorbent particle size, dosage, and transmittance affect the adsorption performance of the low-cost adsorbents. The transmittance parameter was used to assess the performance of the adsorption process in color removal of binary wastewater. NPOC was the parameter used to estimate the organic carbon removal in binary wastewater after the combined treatment of adsorption and microfiltration.

\subsection{Transmittance (\%)}

Transmittance is the parameter used to measure the amount of light that passes through the batch adsorption sample. In the laboratory, using a UV-Visible spectrophotometer, transmittance was measured to assess light's reflectance at $590 \mathrm{~nm}$. To calibrate the spectrophotometer, distilled water was used due to its $(100 \%)$ transmissivity. PAC was the reference adsorbent used to compare the treatment efficiency of low cost adsorbents using transmittance. A comparative bar graph was plotted to understand the adsorbent performance with optimum dosage and size of the low-cost adsorbents and PAC, as shown in Fig. 3. The reference adsorbent PAC treated binary wastewater and improved transmittance to 96.8, 95.3, and $94.2 \%$ in low, medium, and high concentrations. At an optimum dosage of $1.2 \mathrm{~g}$, onion peel seems to get transmittance beyond $80 \%$ at low binary wastewater concentration. At medium and high concentrations, peanut hull seems to get transmittance of 75.9 and $67.4 \%$. Therefore, while onion 
peel seems to perform well at low concentration, peanut hull seems to be a better alternative at medium and high binary wastewater concentrations.

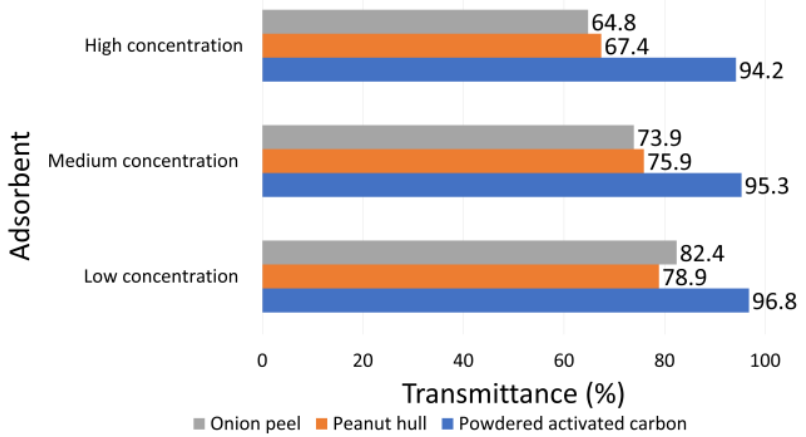

Fig. 3 The transmittance of crystal violet and coffee wastewater at optimum size and dosage

\subsection{NPOC}

NPOC is the amount of organic carbon left after purging out the purgeable carbon using $\mathrm{HCl}$ acid. This parameter was used to assess the change in organic carbon during adsorption and microfiltration treatment by low-cost adsorbents.

\section{0}

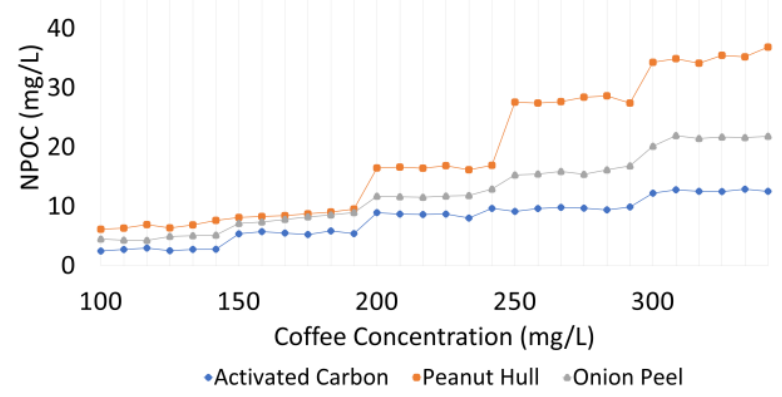

Fig. 4 NPOC of binary wastewater after treatment using adsorbents

Both PAC and onion peel were able to keep the NPOC concentration below $20 \mathrm{mg} / \mathrm{L}$ at varying coffee concentrations in binary wastewater as shown in Fig. 4. Therefore, when compare both the low-cost adsorbents, onion peel seems to perform better than peanut hull in removing organic carbon from binary wastewater.

\subsection{Adsorption Isotherms}

Using beer lambert's law, the final concentration of combined dye and coffee wastewater was estimated. According to beer lambert's law, during equilibrium, the amount of dye adsorbed can be calculated as follows:

$$
q_{e}=\frac{\left(C_{0}-C_{e}\right) V}{W}
$$

where $C_{o}=$ initial concentration $(\mathrm{mg} / \mathrm{L}), C_{e}=$ equilibrium concentration $(\mathrm{mg} / \mathrm{L}), \mathrm{W}=$ Weight of the adsorbent used $(\mathrm{g})$.

The kinetics involved in the adsorption process of $\mathrm{CV}$ and coffee wastewater was investigated using two adsorption isotherms, including Langmuir and Freundlich isotherms. The equations of the isotherms were as follows,

Langmuir isotherm equation, $\frac{1}{q_{e}}=\frac{1}{K_{L} Q_{m}} * \frac{1}{C_{e}}+\frac{1}{Q_{m}}$

Freundlich isotherm equation, $\log q_{e}=\log K_{f}+\frac{1}{n} \log C_{e}$

where $\mathrm{q}_{\mathrm{e}}$ is the uptake, which involves the amount of dye adsorbed at time $\mathrm{t}$ in $\mathrm{mg} / \mathrm{g} . \mathrm{Q}_{\mathrm{m}}$ is the maximum adsorption capacity $(\mathrm{mg} / \mathrm{g}) . \mathrm{K}_{\mathrm{L}}$ is the Langmuir adsorption coefficient, and $\mathrm{K}_{\mathrm{f}}$ is the Freundlich constant for adsorption capacity. $\mathrm{n}$ is the Freundlich constant for intensity. The coefficients of the linearized form of Freundlich and Langmuir isotherms were listed in Table 2.

From Table 2, it was observed that the maximum uptake was 58.14 (mg/g) using onion peel when compared to the peanut hull being 57.47 (mg/g). This uptake shows that onion peel seems to have better adsorption capacity in the case of treating binary wastewater that contains $\mathrm{CV}$ and coffee waste.

Langmuir isotherm seems to have a reasonable fit with $\mathrm{R}^{2}$ of 0.99 using peanut hull compared to using onion peel with $\mathrm{R}^{2}$ being 0.91 as illustrated in Fig. 5. Therefore, the Langmuir isotherm model fits the experimental data better than the Freundlich isotherm model when the peanut hull was used as an adsorbent.

Table 2 Langmuir and Freundlich constants for sorption of binary wastewater using peanut hull and onion peel

\begin{tabular}{lllrrrr}
\hline Adsorbent & Langmuir & \multicolumn{5}{c}{ Freundlich } \\
\cline { 2 - 7 } & $Q_{m}(\mathrm{mg} / \mathrm{g})$ & $K_{L}(\mathrm{~L} / \mathrm{mg})$ & $R^{2}$ & $K_{f}$ & $\mathrm{n}$ & $R^{2}$ \\
\hline Peanut Hull & 57.47 & 0.053 & 0.99 & 0.314 & 0.345 & 0.98 \\
Onion Peel & 58.14 & 0.049 & 0.91 & 1.040 & 1.191 & 0.83 \\
\hline
\end{tabular}

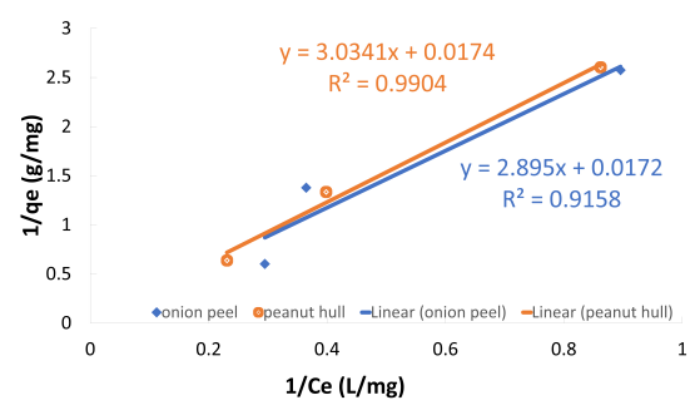

Fig. 5 Langmuir isotherm for binary wastewater using low-cost adsorbents

Similarly, when the Freundlich isotherm model was used to fit the experimental data, binary wastewater treated with peanut hull seems to have a linear relationship when a log transform was used. Experimental concentration data after treatment seems to fit the linear Freundlich model while peanut hull was used in the treatment process with $\mathrm{R}^{2}$ at 0.98 as shown in Fig. 6.

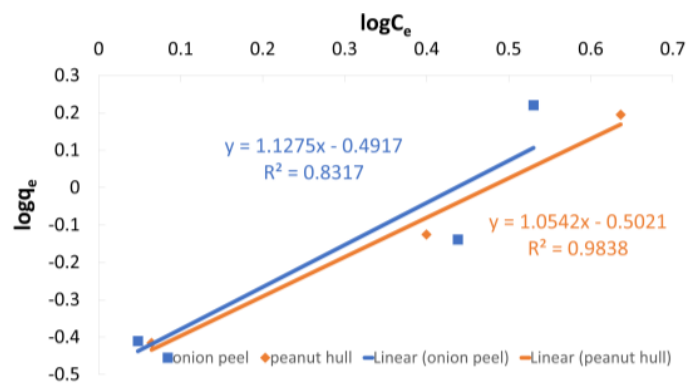

Fig. 6 Freundlich isotherm for binary wastewater using low-cost adsorbents

Since the current study uses binary synthetic wastewater prepared in the laboratory with the temperature at $25^{\circ} \mathrm{C}$ and $1 \mathrm{~atm}$ pressure, the samples were not exposed to oxygen to prevent oxidation. At the industrial scale, the adsorbents used can be further enhanced to achieve higher transmittance results, and the treatment efficiency can vary based on environmental conditions.

\section{Conclusion}

Both the adsorbents, including peanut hull and onion peel, have the potential to treat combined crystal violet and coffee wastewater. Transmittance was the parameter used to assess the color removal of binary wastewater due to combined adsorption and microfiltration processes. Both Langmuir and Freundlich isotherms were used to fit the experimental concentration data of binary wastewater after combined adsorption and microfiltration processes. The linear fit indicates that both Langmuir and Freundlich isotherms follow a linear relationship when peanut hull was used with maximum adsorption capacity being 57.47 $\mathrm{mg} / \mathrm{g}$. In treating binary wastewater using onion peel, both Langmuir and Freundlich isotherms fit the data with the maximum adsorption capacity being $58.14 \mathrm{mg} / \mathrm{g}$.

\section{Acknowledgment}

The authors are grateful to Cleveland State University for providing research facilities. 


\section{References}

[1] F.I. Hai, K. Yamamoto, K. Fukushi, Hybrid treatment systems for dye wastewater, Crit. Rev. Environ. Sci. Technol. 37(4) (2007) 315-377.

[2] F. Mashkoor, A. Nasar, Inamuddin, A.M. Asiri, Exploring the reusability of synthetically contaminated wastewater containing crystal violet dye using tectona grandis sawdust as a very low-cost adsorbent, Sci. Rep. 8(1) (2018) 8314.

[3] S. Chakma, L. Das, V.S. Moholkar, Dye decolorization with hybrid advanced oxidation processes comprising sonolysis/fenton-like/photo-ferrioxalate systems: A mechanistic investigation, Sep. Purif. Technol. 156 (2015) 596-607.

[4] S. Mani, R.N. Bharagava, Exposure to Crystal violet, its toxic, genotoxic and carcinogenic effects on environment and its degradation and detoxification for environmental safety, Rev. Environ. Contam. Toxicol. 237 (2016) 71-104.

[5] C. Allègre, P. Moulin, M. Maisseu, F. Charbit, Treatment and reuse of reactive dyeing effluents, J. Membr. Sci. 269(1) (2006) 15-34.

[6] S.V.H. Madiraju, Y.T. Hung, H.H. Paul, Treatment of disperse blue 14 wastewater and sugar wastewater by low-cost adsorbents, Res J. Chem. Environ. Sci. 6(5) (2018) 16-22.

[7] B.M. Adesanmi, Y.T. hung, H.H. Paul, Coagulation-flocculation treatment for naphthol green band flour wastewater, Int. J. Mod. Trends Sci. Technol. 6(12) (2020) 190-197.

[8] S.S.V. Harsha Madiraju, Yung-Tse Hung, Howard H. Paul, Treatment of acid orange 74 wastewater and sugar wastewater by low-cost adsorbents, J. Adv. Chem. Sci. 4(3) (2018) 583-585.

[9] S.V.H. Madiraju, Color Removal and treatment of dye and sugar waste water using low-cost adsorbents, ETD Arch. 1098, M.Sc. Thesis, Cleveland State University, USA, 2018

[10] A.S.P. Pamula, Adsorption and microfiltration processes to treat dye and coffee wastewater, ETD Arch., 1101, M.Sc. Thesis, Cleveland State University, USA, 2018.

[11] S. Humbert, Y. Loerincik, V. Rossi, M. Margni, O. Jolliet, Life cycle assessment of spray dried soluble coffee and comparison with alternatives (drip filter and capsule espresso), J. Clean. Prod. 17(15) (2009) 1351-1358.
[12] R.K. Sinha, G. Bharambe, P. Bapat, Removal of high BOD and COD loadings of primary liquid waste products from dairy industry by vermifiltration technology using earthworms, Ind. J. Environ. Protect. 27(6) (2007) 486-501.

[13] S.V.H. Madiraju, P.G. Raghunadh, K.R. Kumar, Prototype of eco-friendly indoor air purifier to reduce concentrations of $\mathrm{CO}_{2}, \mathrm{SO}_{2}$ and $\mathrm{NO}_{2}$, Nat. Environ. Pollut. Technol. 19(2) (2020) 747-753.

[14] S.V.H. Madiraju, A. Kumar, L. Nishadhi, Examination of plant-based coagulants to replace lime and alum for surface water treatment, A\&WMA's 112th Annual Conference \& Exhibition, Quebec City, Quebec, Canada, 2019

[15] G. Alaa El-Din, A.A. Amer, G. Malsh, M. Hussein, Study on the use of banana peels for oil spill removal, Alex. Eng. J. 57(3) (2018) 2061-2068.

[16] K. Kenes, O. Yerdos, M. Zulkhair, D. Yerlan, Study on the effectiveness of thermally treated rice husks for petroleum adsorption, J. Non-Cryst. Solids 358(22) (2012) 2964-2969.

17] C. Saka, Ö. Sahin, Removal of methylene blue from aqueous solutions by using cold plasma- and formaldehyde-treated onion skins, Color. Technol. 127(4) (2011) 246-255

[18] C. Saka, Ö. Şahin, M.S. Çelik, The removal of methylene blue from aqueous solutions by using microwave heating and pre-boiling treated onion skins as a new adsorbent, Energy Sources, Part A 34(17) (2012) 1577-1590.

[19] M.A.M. Salleh, D.K. Mahmoud, W.A.W.A. Karim, A. Idris, Cationic and anionic dye adsorption by agricultural solid wastes: A comprehensive review, Desalination 280(1-3) (2011) 1-13.

[20] R. Gong, Y. Ding, M. Li, C. Yang, H. Liu, Y. Sun, Utilization of powdered peanut hull as biosorbent for removal of anionic dyes from aqueous solution, Dyes Pigm. 64(3) (2005) 187-192.

[21] D. Özer, G. Dursun, A. Özer, Methylene blue adsorption from aqueous solution by dehydrated peanut hull, J. Hazard. Mater. 144(1) (2007) 171-179.

[22] K. Anupam, S. Dutta, C. Bhattacharjee, S. Datta, Adsorptive removal of chromium (VI) from aqueous solution over powdered activated carbon: Optimisation through response surface methodology, Chem. Eng. J. 173(1) (2011) 135-143. 